\title{
Agency Costs of Dry Powder in Private Equity Funds
}

Marie Lambert

1. HEC LIÈGE
Alexandre Scivoletto

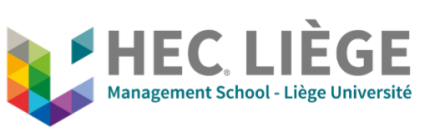

Tereza Tykvová

University of St.Gallen

Research seminar, Toulouse School of Economics, September 21st 2020 


\section{Dry Powder in Private Equity Funds}

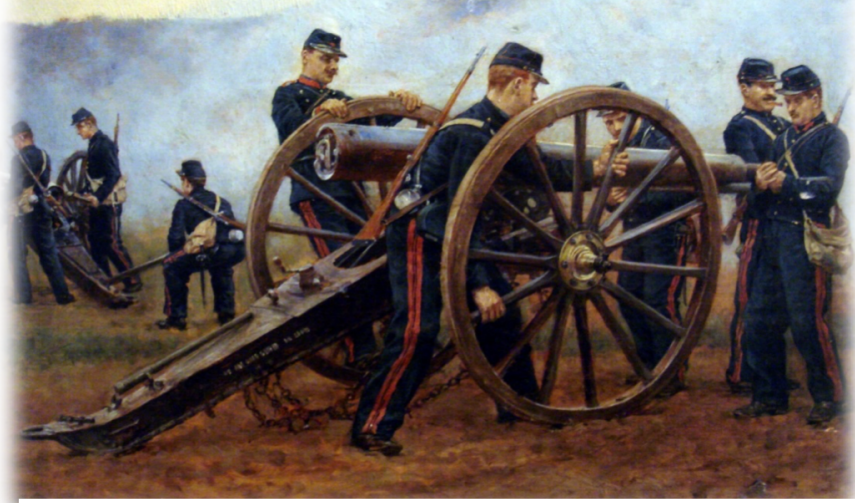

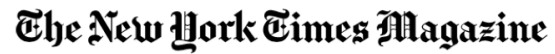

ON LANGUAGE

\section{Keeping Your Powder Dry}

By William Safire
- Gunpowder had to be stored and be dry to be immediately available

- "Keep your powder dry" means "remain calm, keep cool" (NY Times)

- In the context of private equity, dry powder refers to cash still available for investment purposes or "unspent capital", i.e. committed capital by LPs that has not been invested yet 


\section{FORTUNE}

FINANCE • PRIVATE EQUITY

\section{Private equity firms are sitting on $\$ 1.5$ trillion in unspent cash, and looking to raise more}

BY ANNE SRADERS

January 25, 2020 8:00 PM GMT+1

Opinion Private equity

The private equity bubble is bound to burst

There are worrying signs that the sector is becoming a victim of its own success

PATRICK JENKINS

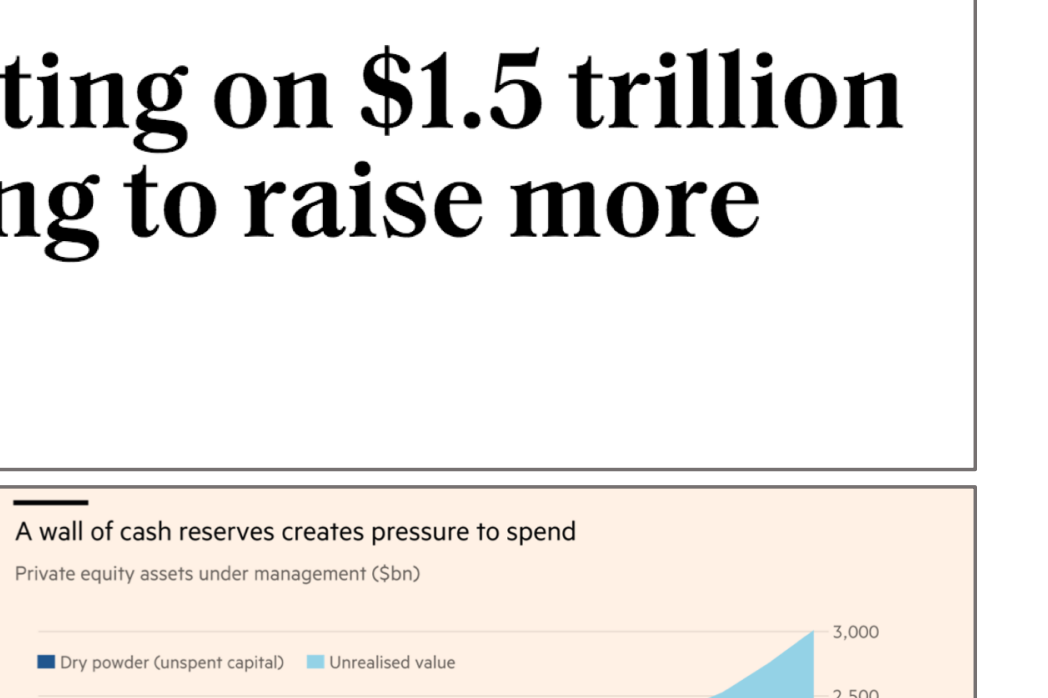

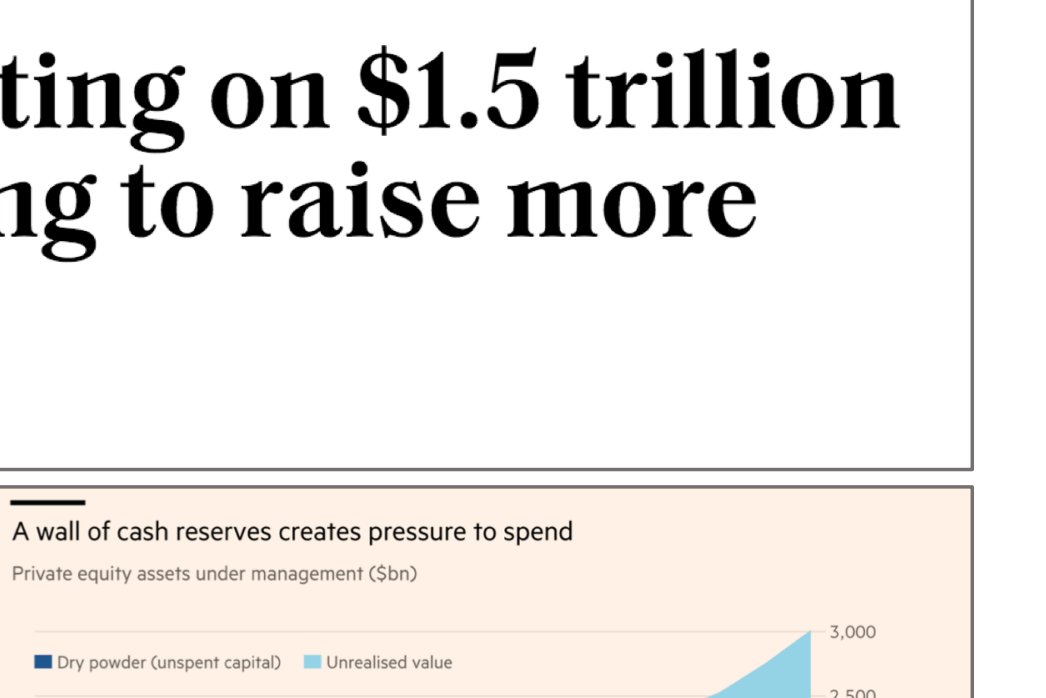

A wall of cash reserves creates pressure to spend

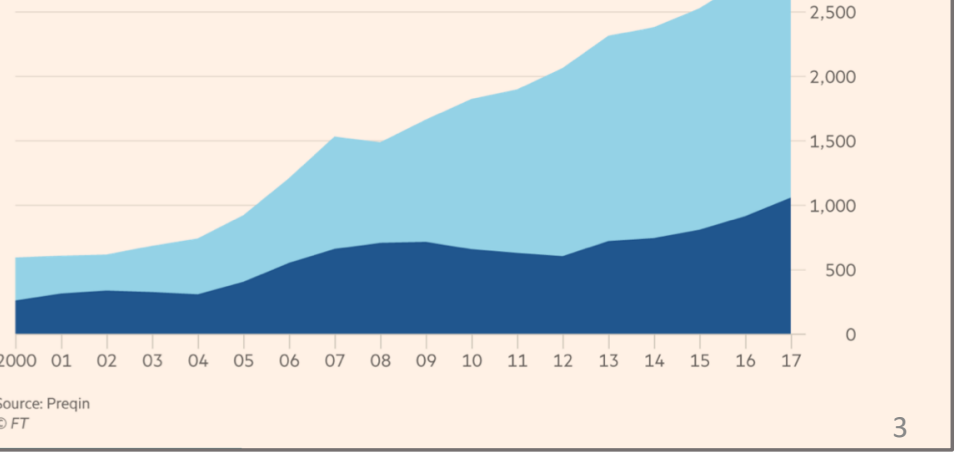




\section{Scope}

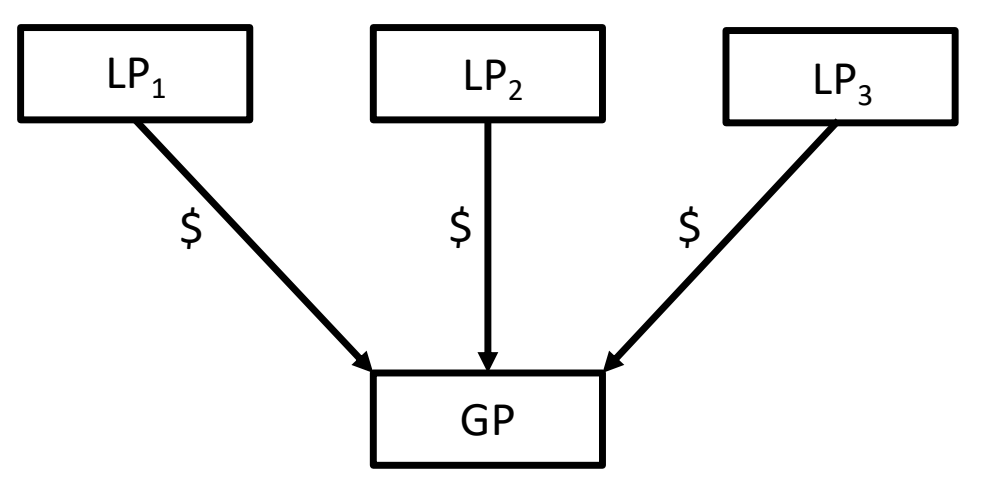

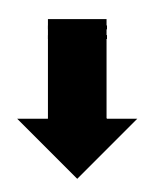

Acquire majority stakes in companies

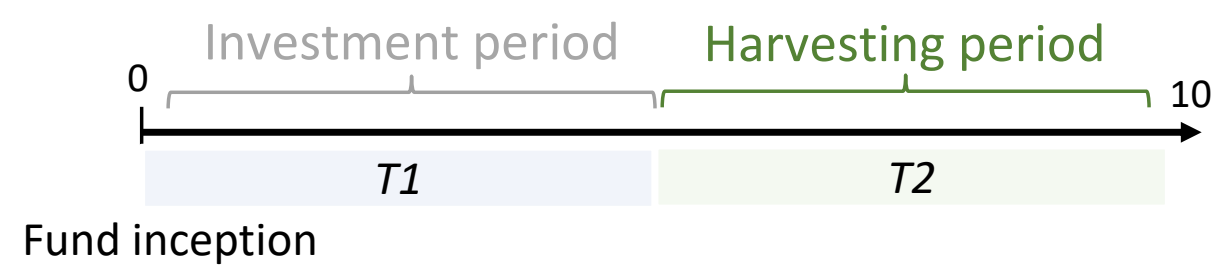

\section{Contract features}

- Fees (GP compensation) : Management fees / Carry interest / Monitoring fees/ Transaction fees

- Hurdle rate

- Catch-up

- Waterfalls

- Clawback 


\section{The paper in a nutshell}

We model GP investment behavior based on their expected fees

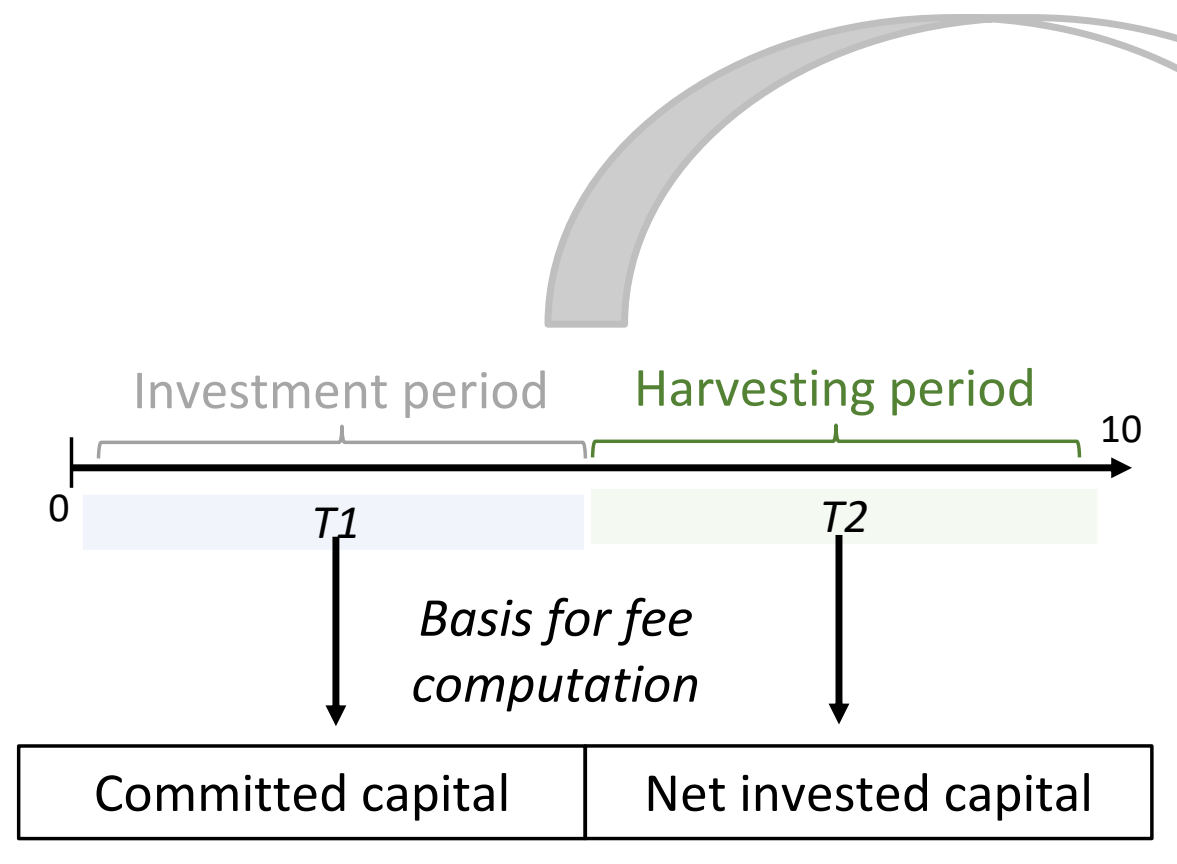

At the end of the investment period

High dry powder

$\rightarrow$ Loss in management fees

$\rightarrow$ Delaying investment to capture better opportunities 


\section{The paper in a nutshell}

We model the GP investment behavior when the basis of fee computation changes

- The expected fees of GP depend on the expected return of the GP, the carry and management fees, exit rate and time to exit. In a first extension, we introduce deal leverage

- Management fees, leverage and expected returns outcome as having a material impact on the dry powder from the revenue management model 


\section{The paper in a nutshell}

We analyze the impact of fees, GP expected return (based on its past performance) on the abnormal level of dry powder at the end of the investment period

- Data: 383 fund sponsoring 1,011 US LBO deals during the period $1980-2019$

- Small funds, funds with low management fees or GP with a weak track record are more likely to have an abnormal level of dry powder at the end of the investing period

- This situation leads to agency costs: We give evidence of loss in performance for funds with abnormal dry powder at the end of the investing period 


\section{The paper in a nutshell}

We examine the characteristics of deals performed at and after vintage year +4

- Data: from $\mathbf{1 0 5}$ to $\mathbf{2 3 0}$ deals on which we have sufficient information (deal terms and exit conditions)

- We find that high levels of dry powder lead to investment distortions where GPs focus more on maximizing their fees rather than maximizing the value for LPs

- Deals undertaken at the end of the investing period by funds with a large volume of dry powder are under-leveraged, are larger and performed with less syndication to maximize the equity spent 


\section{Literature review}

\section{Agency/contract theory}

Jensen \& Meckling (1976, JFE)

Metrick \& Yasuda (2010, RFS)

Axelson et al. (2009, JF); Axelson, et al. $(2013, \mathrm{JF})$

\section{GP experience and reputation}

Gompers (1996, JFE)

Kaplan \& Schoar (2005, JF)

Ljungqvist et al. (2020, FM)

\section{Dry Powder and buying pressure}

Arcot et al. (2015, JFE)

Degeorge et al. (2016, JFE)

\section{PE performance}

Phalippou \& Gottschalg (2009, RFS)

Chung et al. (2012, RFS)

Harris et al. (2014, JF)

Robinson \& Sensoy (2016, JFE) 


\section{Theoretical model}

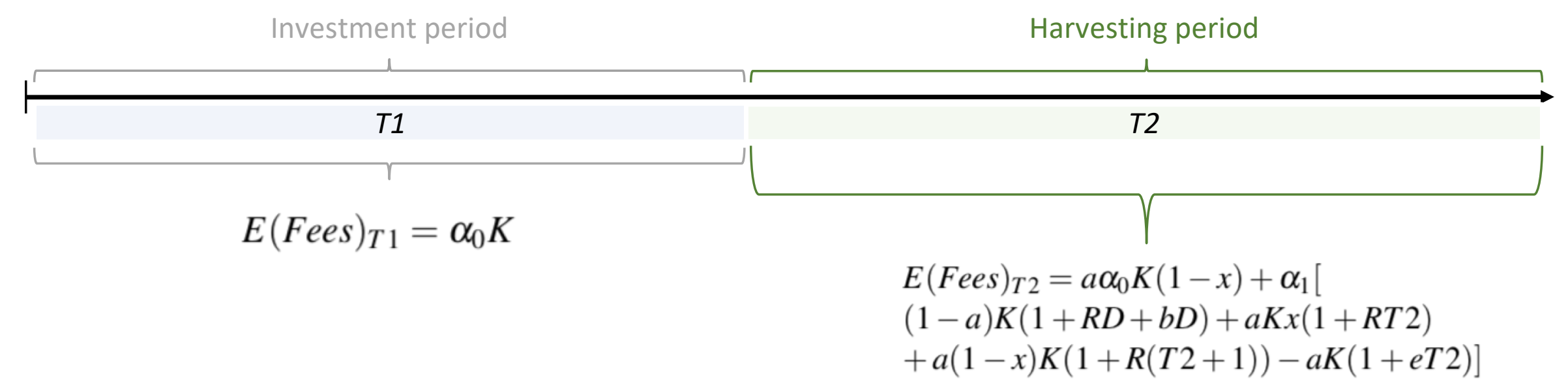

$\underline{\text { Variables }}$

$\alpha_{0}$ : Management fees

$\alpha_{1}$ : Carried interest

e: Hurdle rate
R: Expected return

b: Option to delay

a: \% of invested capital
$\mathrm{K}$ : Total committed capital

T2: Time to exit

$\mathrm{x}$ : Disinvestment rate

D: Duration 


\section{Theoretical model}

\section{Scenario $1(\mathrm{DP}=0$ )}

$$
\begin{aligned}
& \text { E(Fees })_{T 2 \mid S 1}=\alpha_{0} K(1-x)+\alpha_{1}[K x(1+R T 2) \\
& +(1-x) K(1+R(T 2+1))-K(1+e T 2)]
\end{aligned}
$$

Where $\quad \alpha_{0} K(1-x)$

$K x(1+R T 2)$

$(1-x) K(1+R(T 2+1))$

$K(1+e T 2)$
Total management fees at time T2

Cum. perf. of the exited investments

Cum. perf. of the not yet exited investments Min. LP remuneration 


\section{Theoretical model}

\section{Scenario 2 (DP > 0 )}

$$
\begin{aligned}
& E(\text { Fees })_{T 2 \mid S 2}=a \alpha_{0} K(1-x)+\alpha_{1}[ \\
& (1-a) K(1+R D+b D)+a K x(1+R T 2) \\
& +a(1-x) K(1+R(T 2+1))-a K(1+e T 2)]
\end{aligned}
$$

Where $\quad a \alpha_{0} K(1-x)$

$(1-a) K(1+R D+b D)$

$a K x(1+R T 2)$

$a(1-x) K(1+R(T 2+1))$

$a K(1+e T 2)$
Total management fees at time $\mathrm{T} 2$

Potential perf. of the not yet invested capital Cum. perf. of the exited investments

Cum. perf. of the not yet exited investments

Min. LP remuneration 


\section{Theoretical model}

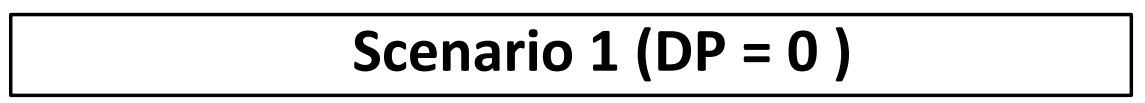

\section{Scenario 1 (DP > 0 )}

$$
\begin{aligned}
& E(\text { Fees })_{T 2 \mid S 1}=\alpha_{0} K(1-x)+\alpha_{1}[K x(1+R T 2) \\
& +(1-x) K(1+R(T 2+1))-K(1+e T 2)]
\end{aligned}
$$

$$
\begin{aligned}
& E(\text { Fees })_{T 2 \mid S 2}=a \alpha_{0} K(1-x)+\alpha_{1}[ \\
& (1-a) K(1+R D+b D)+a K x(1+R T 2) \\
& +a(1-x) K(1+R(T 2+1))-a K(1+e T 2)]
\end{aligned}
$$

\section{Indifference relationship}

$$
\begin{gathered}
\Delta E\left(\text { fees }_{T 2|S 2-T 2| S 1}\right)=0 \\
a=\frac{1}{1+\frac{\alpha_{1}(b D)}{\left.\alpha_{0}(1-x)+\alpha_{1}[R(T 2-x-D)-e T 2)\right]}}
\end{gathered}
$$




\section{Theoretical model}

Fee compensation scheme and DP

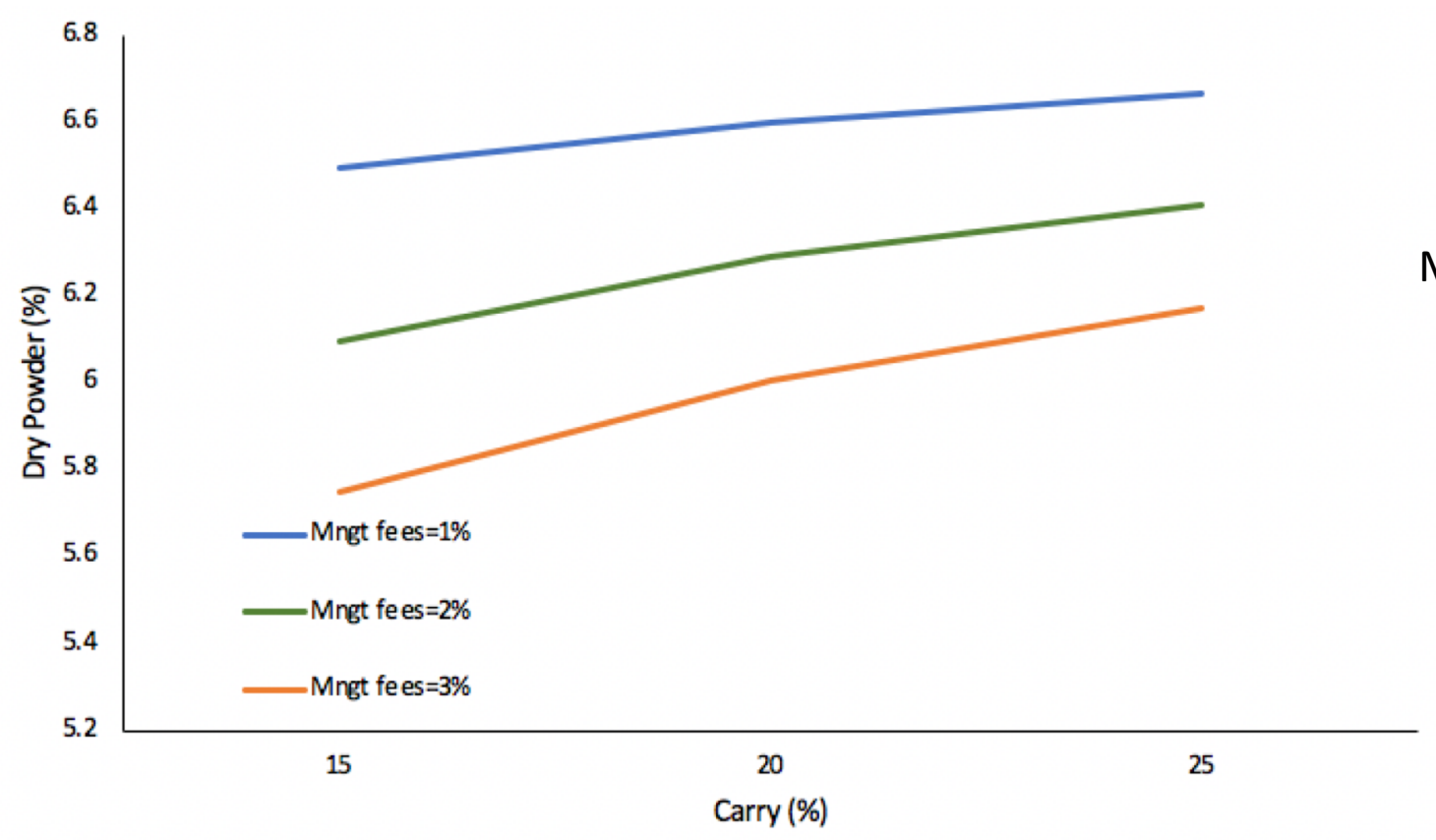

Variation of 100 basis points

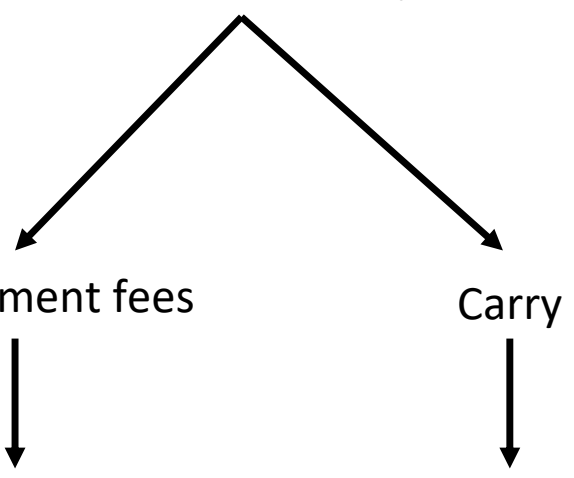

Change in dry powder by 30 basis points
Change in dry powder by 1.6 basis points 


\section{Theoretical model}

\section{GP expected return and DP}

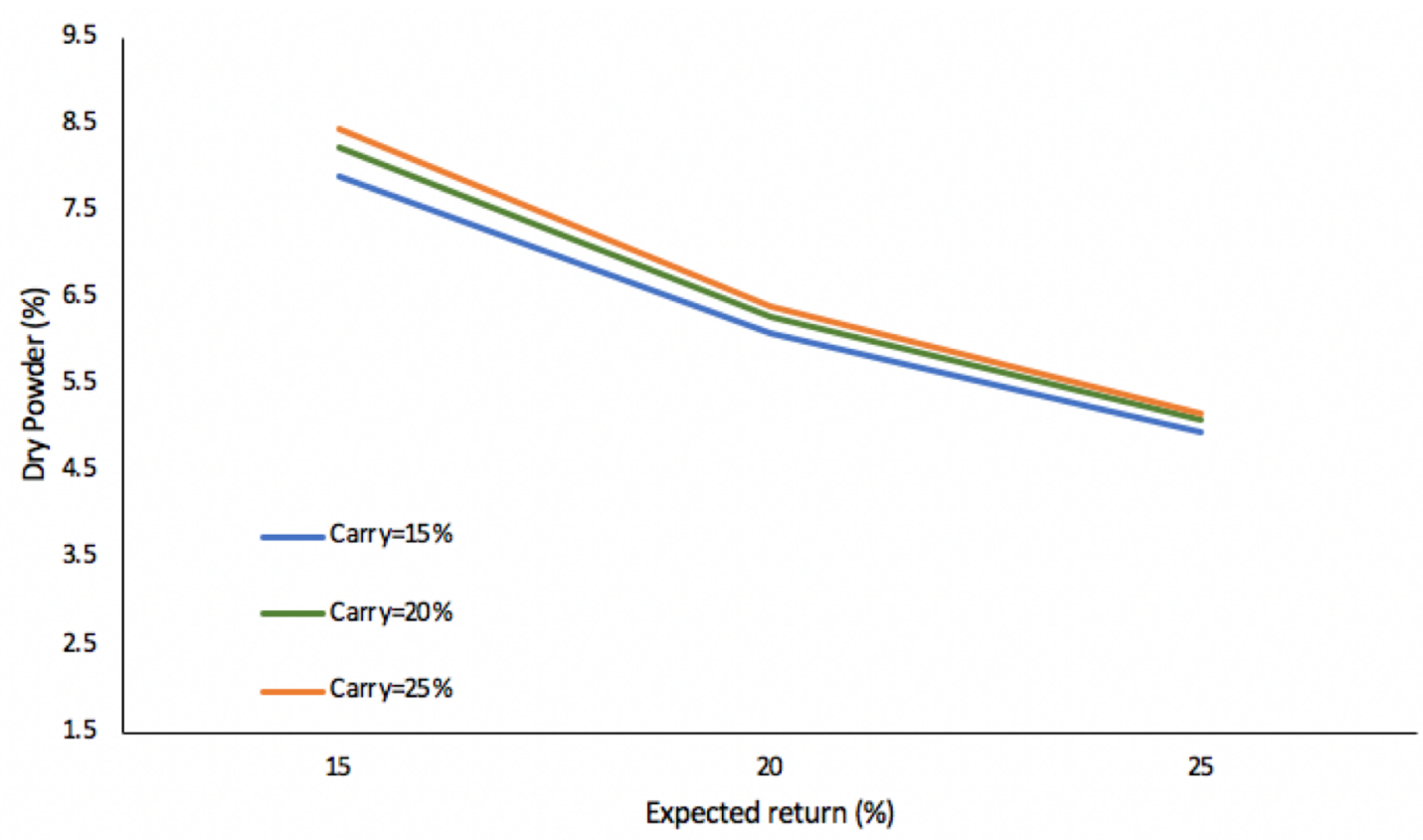

An increase of expected returns of $10 \%$

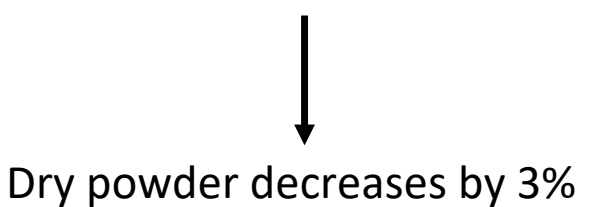




\section{Theoretical model}

\section{Extension 1}

\section{Introducing leverage $L=D / K$}

Deal Value $=a K+a K L$ cost of leverage $=\left(1+r_{0}\right) a K L$

\section{Indifference relationship}

$$
\begin{gathered}
\triangle E\left(\text { fees }_{T 2|S 2-T 2| S 1}\right)=0 \\
L=\frac{(1-a) \alpha_{0}(1-x)+\alpha_{1}[(1-a)(R D+b D)+(a-1)(R(T 2+1-x)-a e T 2)]}{\alpha_{1}\left[(1-a)(R D+b D)+(a-1)\left(R(T 2+1-x)-a r_{0}\right]\right.}
\end{gathered}
$$




\section{Theoretical model}

\section{Extension 1}

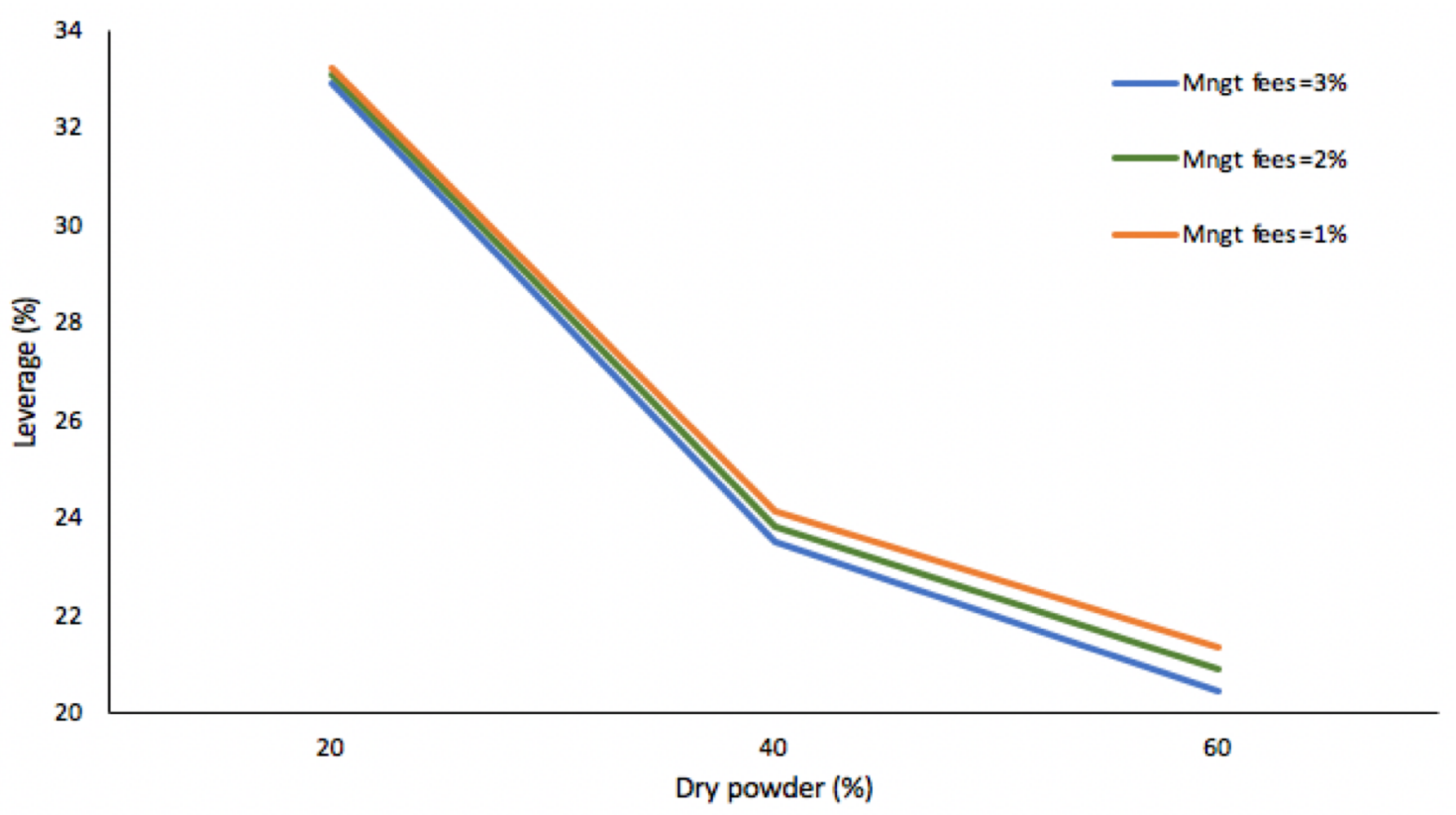

\begin{tabular}{|cc|}
$\uparrow$ Dry powder & $\downarrow$ Leverage \\
$\uparrow$ Mngnt fees & $\downarrow$ Leverage \\
\hline
\end{tabular}




\section{Theoretical model}

\section{Extension 1}

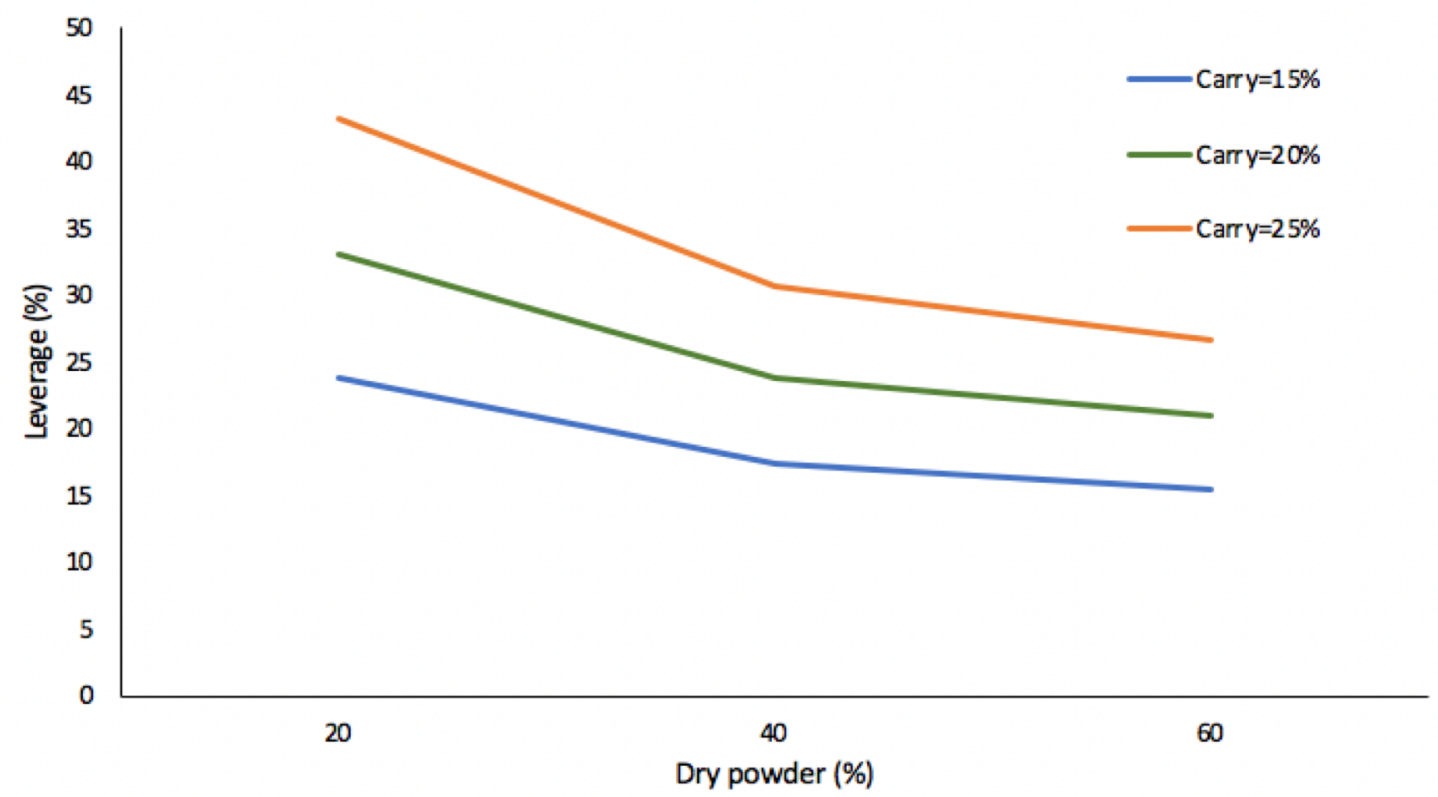

$\begin{array}{ll}\uparrow \text { Dry powder } & \downarrow \text { Leverage } \\ \uparrow \text { Carry } & \uparrow \text { Leverage }\end{array}$




\section{Theoretical model}

\section{Extension 1}

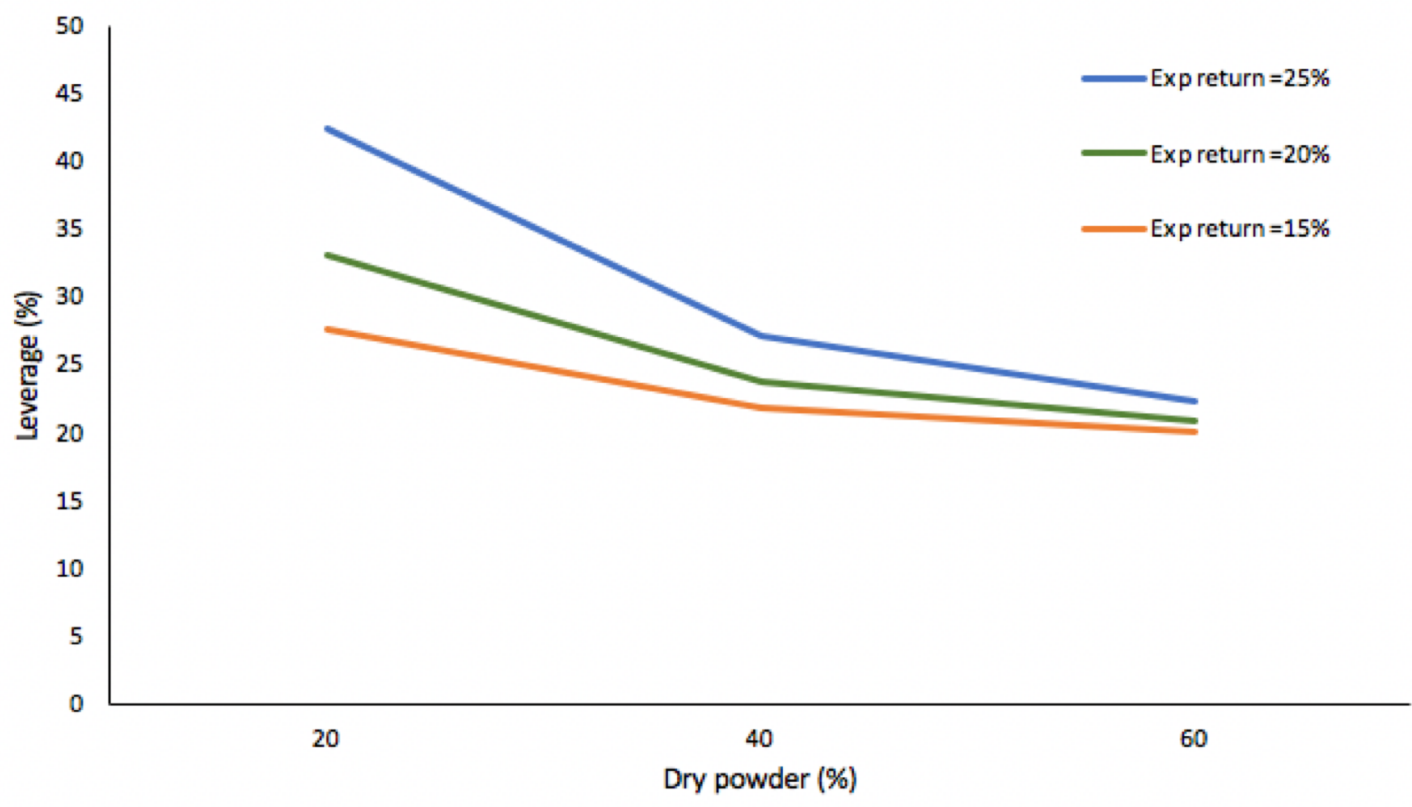

\begin{tabular}{ll}
$\uparrow$ Dry powder & $\downarrow$ Leverage \\
$\uparrow$ Exp returns & $\uparrow$ Leverage \\
\hline
\end{tabular}




\section{Data}

S\&P CIQ

database

EIKON
LBO deals: Going Private Transaction, LBO, MBO,SBO, Platform Deal information: Entry price, entry multiple, target financial metrics, deal syndication

Eikon LBO loans - Adding information on the deal leverage 


\section{Data (continued)}

Private Capital Deals Search Buyout - Adding information on the price/multiple

Private Capital Exits Search Buyout - Adding information on the exit, investment duration, type of exit

Preqin

Private Capital Funds - Adding information on GP characteristics modules (fund number series, fund size, fees structure, fundraising info)

Private Capital Performance - Adding information on GP performance

Private Capital Cash Flow - Adding information on fund cash-flow distributions and dry powder 


\section{Summary statistics (1)}

\begin{tabular}{|c|c|c|c|c|c|c|c|c|c|c|c|c|}
\hline & \multicolumn{4}{|c|}{ Sample 1.2} & \multicolumn{4}{|c|}{ Sample 2.2} & \multicolumn{4}{|c|}{ Sample 1.3} \\
\hline Deal characteristics & Mean & Std. Dev. & $\mathrm{N}$ & $\mathrm{N}$ (funds) & Mean & Std. Dev. & $\mathrm{N}$ & $\mathrm{N}$ (funds) & Mean & Std. Dev. & $\mathrm{N}$ & $\mathrm{N}$ (funds) \\
\hline \multicolumn{13}{|l|}{ Entry characteristics } \\
\hline Entry price (million USD) & 717.79 & 820.96 & 1,011 & 383 & 1035.40 & 880.62 & 578 & 94 & 927.80 & 872.80 & 378 & 65 \\
\hline Leverage of the deal (\%) & & & & & 55.74 & 28.00 & 578 & 94 & & & & \\
\hline Number of funds & 1.41 & 0.79 & 1,011 & 383 & 1.57 & 0.89 & 578 & 94 & 1.57 & 0.85 & 378 & 65 \\
\hline Target revenue (million USD) & 1393.17 & 3483.38 & 532 & 109 & 1749.20 & 3964.42 & 353 & 58 & 1787.73 & 4636.21 & 231 & 39 \\
\hline Target EBITDA margin (\%) & 15.73 & 12.11 & 349 & 63 & 16.41 & 10.91 & 262 & 46 & 17.02 & 11.72 & 162 & 28 \\
\hline Entry revenue multiple & 1.78 & 1.46 & 490 & 79 & 2.06 & 1.56 & 332 & 52 & 2.00 & 1.60 & 215 & 35 \\
\hline Entry EBITDA multiple & 11.75 & 21.45 & 339 & 62 & 11.11 & 17.21 & 256 & 47 & 10.98 & 8.60 & 153 & 27 \\
\hline Target total assets (million USD) & 1069.84 & 1513.82 & 317 & 37 & 1334.10 & 1464.43 & 232 & 38 & 1315.32 & 1500.95 & 317 & 21 \\
\hline \multicolumn{13}{|l|}{ Exit characteristics } \\
\hline Exit price (million USD) & & & & & & & & & 1235.97 & 2585.70 & 378 & 65 \\
\hline Investment duration & & & & & & & & & 4.82 & 2.59 & 378 & 65 \\
\hline Exit multiple & & & & & & & & & 3.39 & 1.77 & 5 & 8 \\
\hline Return (cash multiple) & & & & & & & & & 2.15 & 5.78 & 378 & 65 \\
\hline
\end{tabular}




\section{Summary statistics (2)}

\begin{tabular}{lcll|ll|ll|l|l}
\hline & All Funds & & Q1 Dry Powder Y4 & Q4 Dry Powder Y4 & Diff Test Y4 \\
\hline & Mean & Std. dev. & N & Mean & N & Mean & N & $($ Q1 - Q4) \\
\hline Dry powder Y4 & 0.315 & 0.191 & 383 & 0.071 & 96 & 0.562 & 95 & $(0.492)^{* * *}$ \\
Dry powder Y5 & 0.179 & 0.164 & 383 & 0.014 & 96 & 0.369 & 95 & $(0.354)^{* * *}$ \\
Dry powder Y6 & 0.094 & 0.139 & 383 & -0.017 & 95 & 0.221 & 96 & $(0.238)^{* * *}$ \\
Dry powder change (Y4 to Y6) & 0.221 & 0.138 & 383 & 0.088 & 96 & 0.341 & 95 & $(0.253)^{* * *}$ \\
Fund size (million USD) & 2058.09 & 3173.9 & 383 & 2848.481 & 96 & 1664.596 & 95 & $1183.885^{* * *}$ \\
TVPI & 1.73 & 0.555 & 383 & 1.78 & 96 & 1.72 & 95 & 0.06 \\
KSPME & 1.27 & 0.405 & 383 & 1.34 & 96 & 1.25 & 96 & $0.09^{*}$ \\
Past perf. (TVPI) & 1.90 & 0.610 & 223 & 2.06 & 63 & 1.78 & 53 & $0.276^{* * *}$ \\
Past perf. (KSPME) & 1.277 & 1.337 & 223 & 1.62 & 63 & 1.13 & 53 & $0.49^{*}$ \\
Management fees (\%) & 1.90 & 0.270 & 166 & 1.82 & 39 & 1.91 & 50 & $(0.17)$ \\
Carried interest (\%) & 20.29 & 2.227 & 195 & 20.54 & 44 & 20.55 & 54 & $(0.01)$ \\
GP Fundraising 1 (\%) & 0.32 & 0.470 & 383 & 0.41 & 96 & 0.23 & 96 & $0.18^{* * *}$ \\
First fund (\%) & 0.11 & 0.318 & 383 & 0.13 & 96 & 0.11 & 96 & 0.02 \\
Mid-experienced fund (\%) & 0.39 & 0.490 & 383 & 0.33 & 96 & 0.43 & 96 & $(0.10)^{*}$ \\
Experienced fund (\%) & 0.50 & 0.500 & 383 & 0.53 & 96 & 0.45 & 96 & 0.08 \\
\hline
\end{tabular}




\section{Empirical analysis > Fund level analysis (1)}

$$
\begin{gathered}
y_{4, i}=\alpha_{v}+\beta_{\phi} X_{i}+\gamma_{\mu} Z_{i}+\varepsilon_{i} \\
y_{4, i}=\left\{\begin{array}{ll}
0 & \text { if } D P_{4, i} \leq \operatorname{Median}\left(D P_{4, i}\right) \\
1 & \text { if } D P_{4, i}>\operatorname{Median}\left(D P_{4, i}\right)
\end{array}\right\}
\end{gathered}
$$

- $\mathrm{DP}_{4, i} \quad$ Level of dry powder 4 years after the vintage year of the fund ${ }_{i}$

- $\mathrm{X}_{i} \quad$ Matrix of the ex-ante contract features (management fees, carried interest, exp. returns)

- $\mathrm{Z}_{i} \quad$ Matrix of control variables (fund size, GP experience, GP fundraising, fund risk) 


\section{Empirical results > Fund-level analysis (1)}

Fund / GP features and dry powder (Table 6) - Sample 1.2

\begin{tabular}{|c|c|c|c|c|}
\hline & 1 & 2 & 3 & 4 \\
\hline \multirow[t]{2}{*}{ Infrequent fundraiser } & 0.069 & 0.147 & -0.244 & 0.152 \\
\hline & $(0.152)$ & $(0.385)$ & $(0.463)$ & $(0.547)$ \\
\hline \multirow[t]{2}{*}{ Fund Size } & -0.096 & -0.103 & $-0.450 *$ & $-0.606^{*}$ \\
\hline & $(0.070)$ & $(0.175)$ & $(0.249)$ & $(0.333)$ \\
\hline \multirow[t]{2}{*}{ GP Experience } & -0.016 & -0.133 & $-0.186^{*}$ & $-0.273^{*}$ \\
\hline & $(0.035)$ & $(0.095)$ & $(0.110)$ & $(0.150)$ \\
\hline \multirow[t]{2}{*}{ GP Fundraising 1} & $-0.663 * * *$ & $-1.724 * *$ & $-1.471 *$ & $-1.735^{*}$ \\
\hline & $(0.180)$ & $(0.676)$ & $(0.761)$ & $(0.927)$ \\
\hline \multirow[t]{2}{*}{ Management Fees } & & & $-2.731 * *$ & $-3.334 * *$ \\
\hline & & & $(1.282)$ & $(1.571)$ \\
\hline \multirow[t]{2}{*}{ Carried Interest } & & & -0.042 & 0.013 \\
\hline & & & $(0.116)$ & $(0.148)$ \\
\hline \multirow[t]{2}{*}{ GP Past perf. (TVPI) } & & & & $-1.785 * * *$ \\
\hline & & & & $(0.646)$ \\
\hline \multirow[t]{2}{*}{ Beta_1 } & & & & 0.554 \\
\hline & & & & $(1.478)$ \\
\hline Vintage Year Fixed Effect & Yes & Yes & Yes & Yes \\
\hline $\mathrm{N}$ & 372 & 72 & 72 & 72 \\
\hline Pseudo R-Squared & 0.1005 & 0.2764 & 0.3388 & 0.4606 \\
\hline
\end{tabular}

$86 \%$ of our funds change the basis for fee computations in the harvesting period 


\section{Empirical results > Fund-level analysis (2)}

Fund / GP features and dry powder (Table A.3) - Sample: All closed PE funds (Preqin)

\begin{tabular}{lllll}
\hline & \multicolumn{1}{c}{1} & \multicolumn{1}{c}{2} & \multicolumn{1}{c}{3} & \multicolumn{1}{c}{4} \\
\hline Infrequent fundraiser & $-0.221^{* * *}$ & 0.135 & 0.145 & 0.123 \\
Fund Size & $(0.074)$ & $(0.241)$ & $(0.251)$ & $(0.254)$ \\
& $-0.132^{* * *}$ & -0.107 & -0.207 & -0.202 \\
GP Experience & $(0.070)$ & $(0.108)$ & $(0.128)$ & $(0.129)$ \\
& $0.026^{*}$ & $-0.100^{*}$ & -0.120 & $-0.135^{* *}$ \\
GP Fundraising 1 & $(0.014)$ & $(0.058)$ & $(0.062)$ & $(0.064)$ \\
& $-0.443^{* * *}$ & -0.428 & -0.296 & -0.194 \\
Management Fees & $(0.088)$ & $(0.353)$ & $(0.367)$ & $(0.375)$ \\
& & & $-1.006^{* *}$ & $-1.085^{* * * *}$ \\
Carried Interest & & & $(0.511)$ & $(0.532)$ \\
& & & -0.012 & -0.003 \\
GP Past Perf. & & & $(0.047)$ & $(0.049)$ \\
& & & & -0.251 \\
Beta_1 & & & & $(0.189)$ \\
& & & & 0.881 \\
Vintage Year Fixed Effect & Yes & Yes & Yes & Yes \\
\hline $\mathrm{N}$ & 1374 & 142 & 142 & 142 \\
Pseudo R-Squared & 0.0697 & 0.1452 & 0.1860 & 0.2021 \\
\hline
\end{tabular}




\section{Empirical results > Fund-level analysis (3)}

Fund / GP features and dry powder (Table A.4) - Sample 1.2 - OLS regression

\begin{tabular}{lllll}
\hline & \multicolumn{1}{c}{1} & \multicolumn{1}{c}{2} & \multicolumn{1}{c}{3} & \multicolumn{1}{c}{4} \\
\hline Infrequent fundraiser & 0.006 & 0.033 & 0.021 & 0.033 \\
& $(0.021)$ & $(0.047)$ & $(0.050)$ & $(0.046)$ \\
Fund Size & -0.010 & -0.014 & -0.030 & $-0.042^{*}$ \\
& $(0.009)$ & $(0.021)$ & $(0.024)$ & $(0.224)$ \\
GP Experience & -0.003 & $-0.027^{* *}$ & $-0.027^{* *}$ & $-0.024^{* *}$ \\
& $(0.004)$ & $(0.011)$ & $(0.011)$ & $(0.010)$ \\
GP Fundraising 1 & $-0.091^{* * *}$ & $-0.211^{* * *}$ & $-0.203^{* *}$ & $-0.187^{* *}$ \\
& $(0.024)$ & $(0.071)$ & $(0.078)$ & $(0.072)$ \\
Management Fees & & & -0.144 & $-0.164^{*}$ \\
& & & $(0.098)$ & $(0.090)$ \\
Carried Interest & & & 0.001 & 0.006 \\
& & & $(0.011)$ & $(0.011)$ \\
GP Past Perf. & & & & $-0.135^{* * *}$ \\
& & & & $(0.037)$ \\
Beta_1 & & & & 0.118 \\
& & & & $(0.124)$ \\
\hline Vintage Year Fixed Effect & Yes & Yes & Yes & Yes \\
\hline $\mathrm{N}$ & 372 & 72 & 72 & 72 \\
Pseudo R-Squared & 0.1458 & 0.3957 & 0.4176 & 0.5310 \\
\hline
\end{tabular}




\section{Empirical analysis > Fund level analysis (2)}

$$
\text { Fund performance }{ }_{i}=\alpha_{v}+\beta_{\phi} D P_{4, i}+\gamma_{\mu} Z_{i}+\varepsilon_{i}
$$

- Fund performance ${ }_{i}$ TVPI or KSPME (PE performance measure) of the fund ${ }_{i}$

- $\mathrm{DP}_{4, i}$

- $\mathrm{Z}_{i}$ Level of dry powder 4 years after the vintage year of the fund ${ }_{i}$ Matrix of control variables (fund size, GP experience, GP fundraising)

Vintage year fixed effects included 


\section{Empirical results > Fund-level analysis (4)}

Dry powder and fund sponsor performance (Table 7) -Sample 1.2

\begin{tabular}{lll|ll}
\hline & \multicolumn{2}{c|}{ TVPI } & \multicolumn{2}{c}{ KSPME } \\
\hline & \multicolumn{1}{c}{1} & \multicolumn{1}{c|}{2} & \multicolumn{1}{c}{3} & \multicolumn{1}{c}{4} \\
\hline Dry powder Y4 & & $-0.442^{* *}$ & & $-0.413^{* * *}$ \\
& & $(0.193)$ & & $(0.137)$ \\
Fund size (million USD) & -0.054 & $-0.059^{*}$ & -0.013 & -0.015 \\
& $(0.033)$ & $(0.034)$ & $(0.024)$ & $(0.024)$ \\
GP Experience & $0.029^{*}$ & 0.025 & 0.014 & 0.009 \\
& $(0.017)$ & $(0.017)$ & $(0.012)$ & $(0.012)$ \\
GP Past Perf. & $0.158^{* *}$ & $0.133^{* *}$ & 0.015 & 0.031 \\
& $(0.061)$ & $(0.060)$ & $(0.037)$ & $(0.037)$ \\
\hline Vintage year fixed effect & Yes & Yes & Yes & Yes \\
\hline $\mathrm{N}$ & 223 & 223 & 223 & 223 \\
R-Squared & 0.1893 & 0.2098 & 0.1626 & 0.1985 \\
\hline
\end{tabular}




\section{Empirical results > Fund-level analysis (5)}

Dry powder and fund sponsor performance (Table A.5) - Sample: All closed PE funds (Preqin).

\begin{tabular}{|c|c|c|c|c|}
\hline & \multicolumn{2}{|c|}{ TVPI } & \multicolumn{2}{|c|}{ KSPME } \\
\hline & 1 & 2 & 3 & 4 \\
\hline Dry powder Y4 & & $\begin{array}{l}-0.253^{* *} * \\
(0.106)\end{array}$ & & $\begin{array}{l}-0.159 * * \\
(0.069)\end{array}$ \\
\hline Fund size & $\begin{array}{l}-0.014 \\
(0.016)\end{array}$ & $\begin{array}{l}-0.020 \\
(0.016)\end{array}$ & $\begin{array}{l}-0.003 \\
(0.010)\end{array}$ & $\begin{array}{l}-0.001 \\
(0.010)\end{array}$ \\
\hline GP Experience & $\begin{array}{l}0.016^{* * *} \\
(0.007)\end{array}$ & $\begin{array}{l}0.017^{*} * * \\
(0.007)\end{array}$ & $\begin{array}{l}0.008 * \\
(0.004)\end{array}$ & $\begin{array}{l}0.008 * \\
(0.005)\end{array}$ \\
\hline GP Past Perf. & $\begin{array}{l}0.384 * * * \\
(0.028)\end{array}$ & $\begin{array}{l}0.375^{* * * *} \\
(0.028)\end{array}$ & $\begin{array}{l}0.381 \text { *** } \\
(0.025)\end{array}$ & $\begin{array}{l}0.373 \\
(0.026)\end{array}$ \\
\hline Vintage year fixed effect & Yes & Yes & Yes & Yes \\
\hline $\mathrm{N}$ & 893 & 893 & 893 & 893 \\
\hline R-Squared & 0.2129 & 0.2179 & 0.2204 & 0.2243 \\
\hline
\end{tabular}




\section{Empirical analysis $>$ Deal level analysis}

$$
\mathrm{X}_{d}=\alpha_{v}+\beta_{\phi} D P_{d}+\gamma_{1} Z_{d}+\gamma_{2} Z_{d, f}+\varepsilon_{d}
$$

- $\mathrm{X}_{d} \quad$ Dependent variable (cash return, log deal size*, entry multiple, leverage, deal syndication)

- $\mathrm{DP}_{d} \quad$ Level of dry powder one quarter before the deal initiation

- $\mathrm{Z}_{d} \quad$ Matrix of deal control variable (log deal size -except for equation*)

- $\mathrm{Z}_{d, f}$ Matrix of fund sponsor control variables (fund size, GP experience, GP fundraising, GP past performance) 


\section{Empirical results $>$ Deal-level analysis (1)}

Dry powder and deal size (Table 10) - Sample 1.2

\begin{tabular}{|c|c|c|c|c|}
\hline & \multicolumn{4}{|c|}{ Deal Investment Year $>=$ Vintage year +4} \\
\hline & 1 & 2 & 3 & 4 \\
\hline \multirow[t]{2}{*}{ DP } & 0.526 & $0.954 * *$ & $0.976^{* *}$ & $1.706^{* *}$ \\
\hline & $(0.527)$ & $(0.445)$ & $(0.446)$ & $(0.758)$ \\
\hline \multirow[t]{2}{*}{ Fund size } & & $0.769 * * *$ & $0.765 * * *$ & $0.787 * * *$ \\
\hline & & $(0.085)$ & $(0.087)$ & $(0.132)$ \\
\hline \multirow[t]{2}{*}{ GP Experience } & & $-0.067 *$ & $-0.070^{*}$ & -0.088 \\
\hline & & $(0.040)$ & $(0.040)$ & $(0.057)$ \\
\hline \multirow[t]{2}{*}{ GP Fundraising 2} & & & 0.178 & 0.018 \\
\hline & & & $(0.212)$ & $(0.311)$ \\
\hline \multirow[t]{2}{*}{ GP Past Perf. } & & & & -0.104 \\
\hline & & & & $(0.285)$ \\
\hline Industry Fixed Effect & Yes & Yes & Yes & Yes \\
\hline Investment Year Fixed Effect & Yes & Yes & Yes & Yes \\
\hline $\mathrm{N}$ & 230 & 230 & 230 & 230 \\
\hline R-Squared & 0.1058 & 0.3775 & 0.3797 & 0.4651 \\
\hline
\end{tabular}




\section{Empirical results $>$ Deal-level analysis (2)}

Dry powder and cash on cash return (Table 9) - Sample 1.3

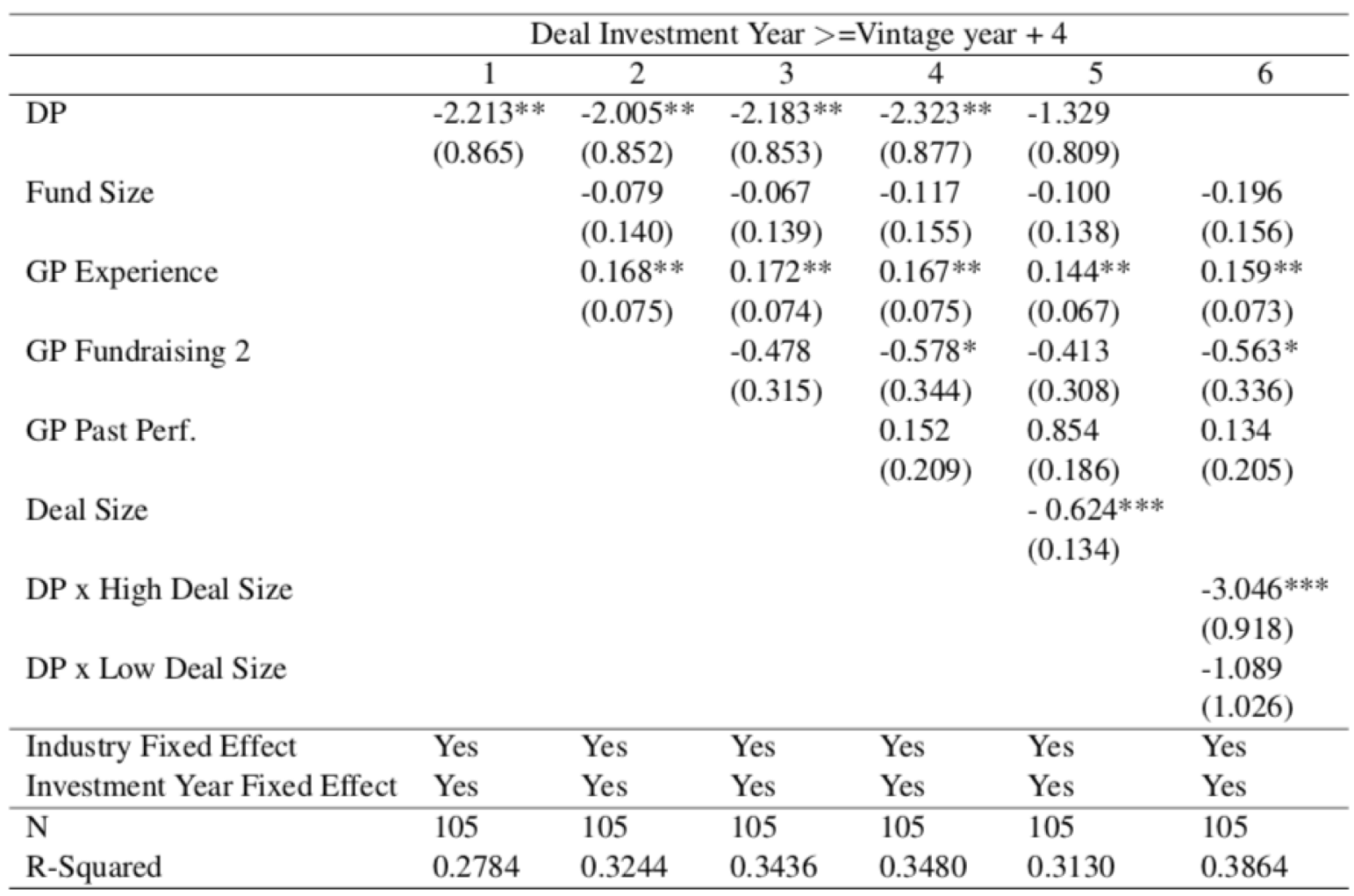




\section{Empirical results $>$ Deal-level analysis (3)}

Dry powder and cash on cash return (Table A.6) - Preqin databases (deals and fund info)

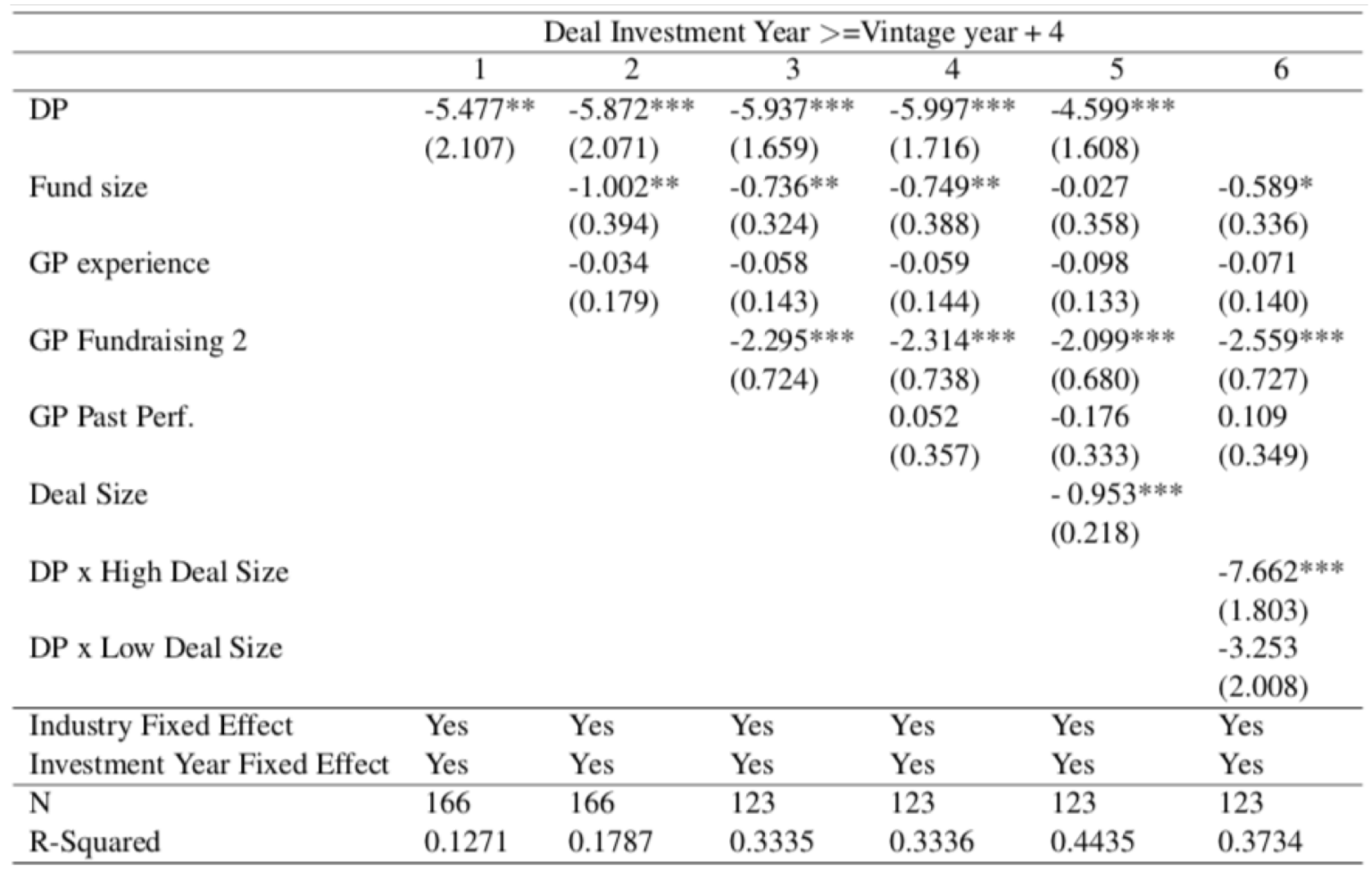




\section{Empirical results $>$ Deal-level analysis (4)}

Dry powder and deal pricing (Table 11) - Sample 1.2

\begin{tabular}{|c|c|c|c|c|c|c|}
\hline \multicolumn{7}{|c|}{ Deal Investment Year $>=$ Vintage year +4} \\
\hline & 1 & 2 & 3 & 4 & 5 & 6 \\
\hline DP & $\begin{array}{l}3.771 \\
(3.612)\end{array}$ & $\begin{array}{l}4.047 \\
(3.525)\end{array}$ & $\begin{array}{l}4.418 \\
(3.552)\end{array}$ & $\begin{array}{l}3.900 \\
(3.691)\end{array}$ & $\begin{array}{l}3.858 \\
(3.725)\end{array}$ & \\
\hline Fund size & & $\begin{array}{l}-1.559 * \\
(0.853)\end{array}$ & $\begin{array}{l}-1.506 * \\
(0.855)\end{array}$ & $\begin{array}{l}-1.046 \\
(0.871)\end{array}$ & $\begin{array}{l}-1.373 \\
(0.972)\end{array}$ & $\begin{array}{l}-1.207 \\
(0.910)\end{array}$ \\
\hline GP experience & & $\begin{array}{l}0.895 * * \\
(0.367)\end{array}$ & $\begin{array}{l}0.919 * * \\
(0.369)\end{array}$ & $\begin{array}{l}0.989 * * \\
(0.392)\end{array}$ & $\begin{array}{l}0.990 * * \\
(0.394)\end{array}$ & $\begin{array}{l}0.959 * * \\
(0.394)\end{array}$ \\
\hline GP Fundraising 2 & & & $\begin{array}{l}-1.862 \\
(2.050)\end{array}$ & $\begin{array}{l}-1.709 \\
(2.077)\end{array}$ & $\begin{array}{l}-1.643 \\
(2.147)\end{array}$ & $\begin{array}{l}-1.614 \\
(2.089)\end{array}$ \\
\hline GP Past Perf. & & & & $\begin{array}{l}-0.507 \\
(0.933)\end{array}$ & $\begin{array}{l}-0.498 \\
(0.941)\end{array}$ & $\begin{array}{l}-0.494 \\
(0.935)\end{array}$ \\
\hline Deal Size & & & & & $\begin{array}{l}-0.099 \\
(0.757)\end{array}$ & \\
\hline DP x High Deal Size & & & & & & $\begin{array}{l}8.122 \\
(6.174)\end{array}$ \\
\hline DP x Low Deal Size & & & & & & $\begin{array}{l}3.786 \\
(3.699)\end{array}$ \\
\hline Industry Fixed Effect & Yes & Yes & Yes & Yes & Yes & Yes \\
\hline Investment Year Fixed Effect & Yes & Yes & Yes & Yes & Yes & Yes \\
\hline $\mathrm{N}$ & 120 & 120 & 120 & 120 & 120 & 120 \\
\hline R-Squared & 0.1620 & 0.2290 & 0.2358 & 0.2383 & 0.2384 & 0.2444 \\
\hline
\end{tabular}




\section{Empirical results $>$ Deal-level analysis (5)}

Dry powder and leverage (Table 12) - Sample 2.2

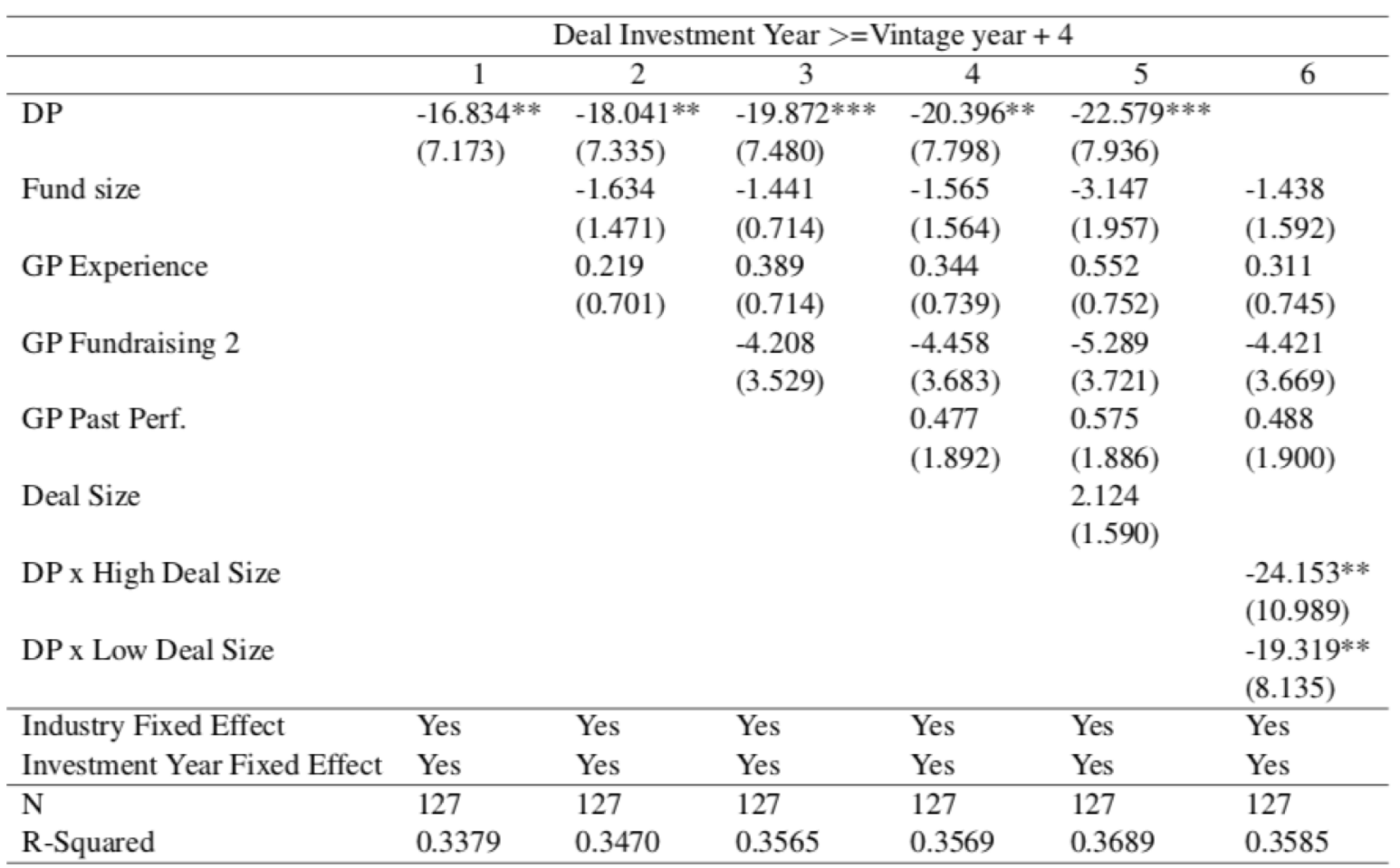




\section{Empirical results $>$ Deal-level analysis (6)}

Dry powder and deal syndication (Table 13) - Sample 1.2

\begin{tabular}{llllll}
\hline \multicolumn{5}{c}{} & \multicolumn{5}{c}{ Deal Investment Year $>=$ Vintage year +4} \\
\hline DP & \multicolumn{1}{c}{1} & \multicolumn{1}{c}{3} & \multicolumn{1}{c}{4} & \multicolumn{1}{c}{5} \\
Fund size & -0.211 & $-0.239^{*}$ & $-0.248^{*}$ & $-0.248^{*}$ & $-0.242^{*}$ \\
& $(0.144)$ & $(0.143)$ & $(0.145)$ & $(0.145)$ & $(0.145)$ \\
GP Experience & & $-0.074^{* * *}$ & $-0.074 * * *$ & $-0.009 * * *$ & $-0.070^{* *}$ \\
& & $(0.027)$ & $(0.027)$ & $(0.028)$ & $(0.028)$ \\
GP Fundraising 1 & & 0.008 & 0.009 & 0.009 & 0.009 \\
& & $(0.014)$ & $(0.014)$ & $(0.015)$ & $(0.015)$ \\
GP Past Perf. & & & -0.033 & -0.035 & -0.035 \\
& & & $(0.076)$ & $(0.078)$ & $(0.078)$ \\
Deal Size & & & & 0.004 & 0.002 \\
& & & & $(0.035)$ & $(0.034)$ \\
Industry Fixed Effect & Yes & & & & $0.047 * *$ \\
Investment Year Fixed Effect & Yes & Yes & Yes & Yes & Yes \\
\hline N & 230 & 230 & 230 & 230 & 230 \\
R-Squared & 0.1155 & 0.1286 & 0.1289 & 0.1289 & 0.1364 \\
\hline
\end{tabular}




\section{Concluding remarks}

- Policy making

- Design of optimal contract between GP and LPs: Focus on management fee and fee computation basis

- New insight about the efficiency of GP-LPs contract terms: GP objective to maximize value creation for LP versus objective maximize the fee collection

- Next steps

- Merge with other datasets to increase observations

- Improve theoretical modelling

- Work on you feedback $:$ (very first presentation) 
Thanks a lot for your attention ! 